\title{
Fabrication of Novel Structures on Silicon with Femtosecond Laser Pulses
}

\author{
W.C. Shen ${ }^{1}$, C.W. Cheng ${ }^{1 *}$, M.C. Yang ${ }^{1}$, Y. Kozawa ${ }^{2}$, S. Sato ${ }^{2 *}$ \\ ${ }^{* 1}$ ITRI South, Industrial Technology Research Institute, No. 8, Gongyan Rd., Liujia Shiang, Tainan \\ County 734, Taiwan, R.O.C. \\ E-mail: CWCheng@itri.org.tw (C.W. Cheng) \\ ${ }^{* 2}$ Institute of Multidisciplinary Research for Advanced Materials, Tohoku University, Katahira 2-1- \\ 1, Aoba-ku, Sendai 980-8577, Japan \\ E-mail: sato@tagen.tohoku.ac.jp.(S.Sato)
}

In this study, the fabrication of novel micro/nano structures were induced on silicon surfaces using radially and azimuthally polarized femtosecond laser, with a $\sim 120$ fs pulse duration, an $800 \mathrm{~nm}$ wavelength, and a $1 \mathrm{kHz}$ repetition rate. Results showed that, as the laser fluence approached the ablation threshold of the silicon, nanostructures with a period of approximately $600 \sim 700 \mathrm{~nm}$ were formed at the low laser fluence region $\left(0.7 \sim 0.8 \mathrm{~J} / \mathrm{cm}^{2}\right)$, and their orientations were perpendicular to the laser polarization. However, as the laser fluence increased $\left(0.9 \sim 1.1 \mathrm{~J} / \mathrm{cm}^{2}\right)$, microstructures with a period of approximately $2 \sim 3 \mu \mathrm{m}$ were formed, and their orientation were parallel to the laser polarization.

DOI:10.2961/jlmn.2010.03.0009

Keywords: femtosecond laser, radial polarization, azimuthal polarization, nanostructure

\section{Introduction}

Recently, the femtosecond laser has been shown as effective for surface micro/nano modification due to its minimal thermal and mechanical damages on silicon and other materials. When irradiating silicon with single-beam femtosecond laser pulses near the ablation threshold, the formation of surface structures, such as ripples (periodic nanostructures) or self-organized micro structures, are observed [1-12]. The formation of periodic nanostructures may be attributed to the actions of the optical interference of an incident femtosecond laser irradiation, with a surface scattered wave [8] for silicon and two plasmon decay models [13-14] for metal material.

However, most research is limited to the linear or circular polarizations of the femtosecond laser beam. This study presented the fabrication of novel periodic nanostructures and microstructures, using radially and azimuthally polarized femtosecond laser, with an $800 \mathrm{~nm}$ wavelength, and a $1 \mathrm{kHz}$ repetition rate. The results showed that periodic nanostructures, with a period of approximately $600 \sim 700 \mathrm{~nm}$, were formed at the low laser fluence region, and periodiclike microstructures, with a period of around $2 \sim 3 \mu \mathrm{m}$, were formed at the high laser fluence region. The formation of periodic nanostructures was consistent with the results reported by previous studies $[2,6,10,11]$ that used linearly polarized femtosecond laser beam. However, to our knowledge, no reports have shown the formation of periodic-like micrometer structures with femtosecond laser irradiation.

\section{Experimental setup}

Figure 1 presents a schematic illustration of the experimental setup, where a polished 4-inch p-type Si (100) wafer with thickness of $200 \mu \mathrm{m}$ was machined in air using a commercial regenerative amplified mode-locked Ti:Sapphire laser (SPIT FIRE, Spectra-Physics), with a central wavelength of $800 \mathrm{~nm}$, a repetition rate of $1 \mathrm{kHz}$, a pulse duration of $\sim 120 \mathrm{fs}$, and a maximum pulse energy of $3.5 \mathrm{~mJ}$. As shown, a pinhole with an aperture of $0.25 \mathrm{~mm}$ was installed in the optical path to improve beam quality. The polarized Gaussian laser beam was attenuated initially using a half-wave plate, and was then incident upon a polarizer, specifically, a polarizing beam splitter. The laser beam passed through an axisymmetric polarization element (SWP-780, Photonic Lattice, Inc.), and the polarization was changed to radial or azimuthal polarization through appropriately segmented waveplates [15].

The intensity beam profile produced by a segmented waveplate was modified into a doughnut-like shape, as shown in Fig. 2. The duodecimal intensity pattern, in addition to the doughnut pattern, was attributed to the polarization element that contains 12 sectors of half-wave plate with different axial directions. Moreover, the polarization pattern was radial and azimuthal, as analyzed by a linear polarizer. Meanwhile, the transmitted laser beam passed through a reflective mirror system to enter the objective lens (numerical aperture 0.14, M Plan Apo NIR, Mitutoyo, magnification $5 \mathrm{x}$ ), and was incident in the normal direction on the surface of a specimen, which was mounted on an X$\mathrm{Y}$ axial micro-positioning stage under a precision greater than $1 \mu \mathrm{m}$. The position of the objective lens was adjusted in the vertical (i.e. Z-axis) direction.

For better observation of the induced structures and in order to reduce the diffraction effect, the laser beam was focused at a position of $300 \mu \mathrm{m}$ below the surface of the $\mathrm{Si}$, and the diameter of the laser spot size at the sample surface was approximately $90 \mu \mathrm{m}$. The surface characteristics were then observed using a scanning electron microscopy (SEM), and the period of structures was the distance between two neighboring structures estimated from the SEM image. 


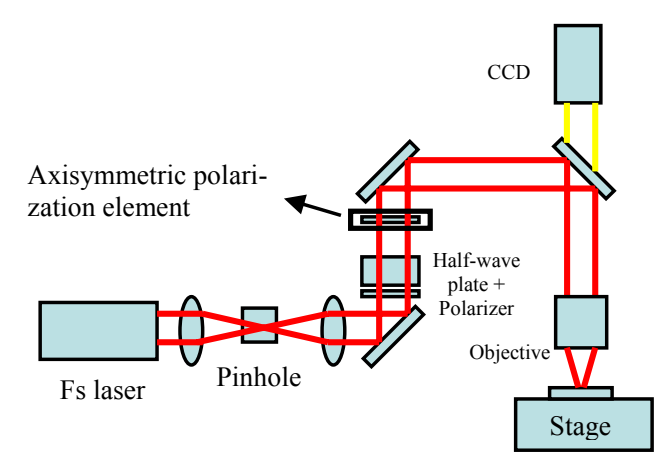

Fig. 1 Schematic illustration showing experimental setup

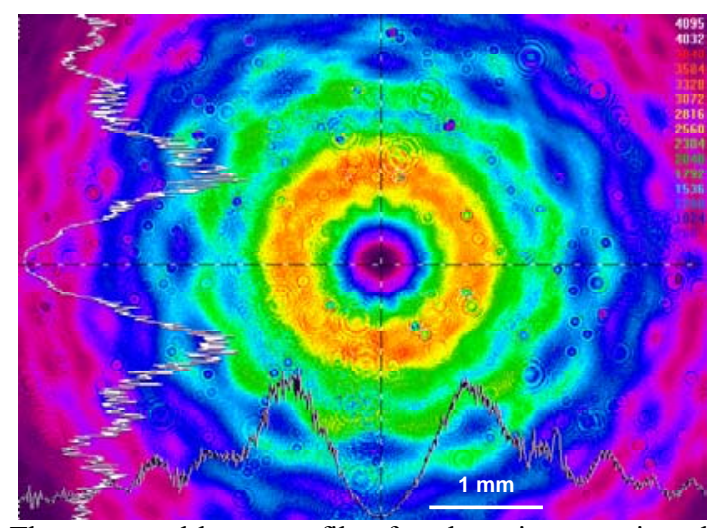

Fig. 2 The measured beam profile after the axisymmetric polarization elements

\section{Results and Discussion}

Figure 3 presents the SEM image of the induced pattern irradiated by azimuthally and radially polarized femtosecond laser beam. In each case, the number of irradiated laser pulses was 200 shots, and the average laser fluence was $0.84 \mathrm{~J} / \mathrm{cm}^{2}$. The laser fluence was slightly higher than the single-shot ablation threshold of the silicon at $0.637 \mathrm{~J} / \mathrm{cm}^{2}$ [16]. Since the irradiation intensity of the laser beam had a donut-shape distribution (see Fig. 2), the center area was subject to a lower energy input. As seen, no obvious surface modification occurred, and only a little debris was observed. However, the outer ring was subject to a higher energy input, and thus, the regions of the micro/nano structures were found after laser irradiation.

Figures 4 5 present the magnified images of areas A and $B$ that are shown in Figs. 3(a) (b), respectively. As seen, nanostructures with a period of approximately 600 700 nm were formed at the low laser fluence region $\left(0.7 \sim 0.8 \mathrm{~J} / \mathrm{cm}^{2}\right)$, which was slightly less than a laser wavelength of $800 \mathrm{~nm}$. In addition, the orientations of the periodic nanostructures were nearly perpendicular to the polarization direction in all cases. The formation of the periodic nanostructures was consistent with the results reported by previous studies $[2,6,10,11]$ that used linearly polarized femtosecond laser beam. Disordered microstructures, with a period of around $2 \sim 3 \mu \mathrm{m}$, were formed at the higher laser fluence region $\left(0.9 \sim 1.1 \mathrm{~J} / \mathrm{cm}^{2}\right)$.

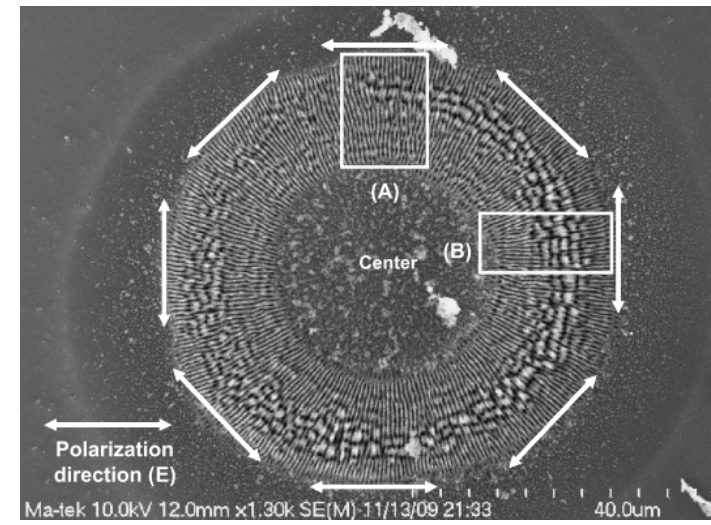

(a)

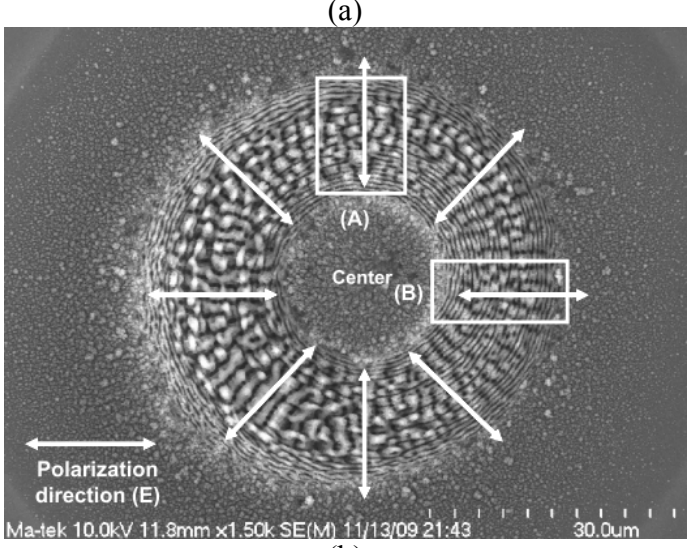

(b)

Fig. 3 SEM images of point pattern irradiated by: (a) azimuthally and (b) radially polarized femtosecond laser beam, respectively. The number of irradiated laser pulses was 200 shots, and the laser fluence was $0.84 \mathrm{~J} / \mathrm{cm}^{2}$ in all cases.

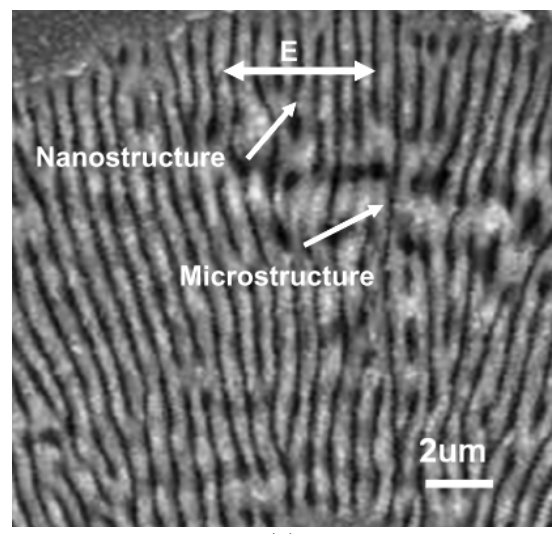

(a)

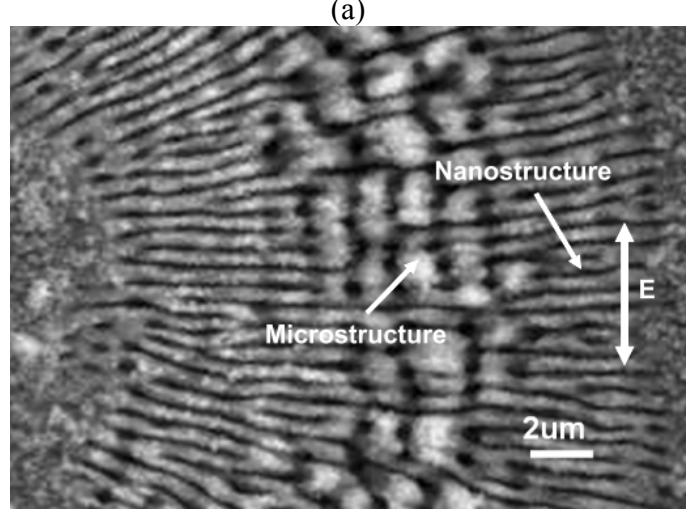

(b)

Fig. 4 Magnified SEM images of Fig. 3(a): (a) area A, and (b) area $\mathrm{B}$ 


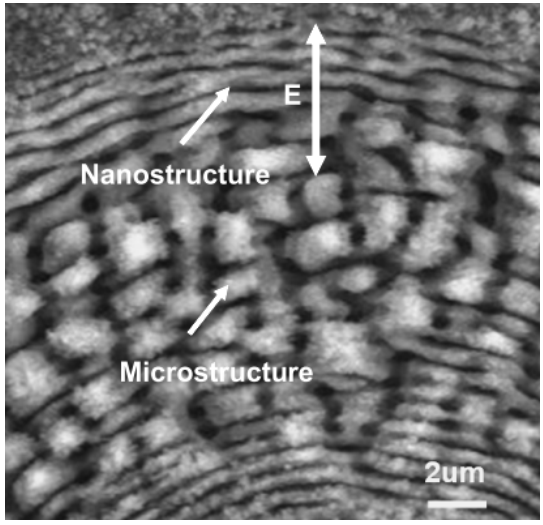

(a)

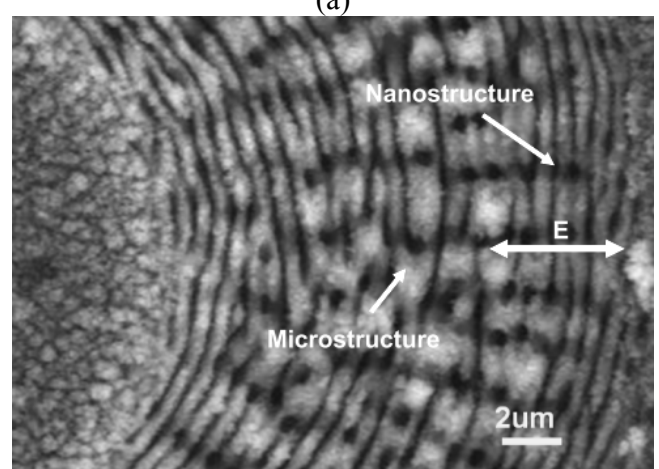

(b)

Fig. 5 Magnified SEM images of Fig. 3(b): (a) area A, and (b) area B.

Figures 6 and 7 present the magnified SEM images of the point pattern irradiated at azimuthally and radially polarized femtosecond laser beam, respectively. The number of irradiated laser pulses was 200 shots, and the laser fluence was in the range of $0.94 \sim 1.01 \mathrm{~J} / \mathrm{cm}^{2}$. As the laser fluence increased, micrometer areas became larger. Micrometer periodic-like structures were observed at the high laser fluence region, and the periodic nanostructures were still at the lower fluence region. In addition, the orientations of the micrometer periodic-like structures were approximately parallel to the polarization direction, while the periodic nanostructures remained perpendicular to the polarization direction. In other words, the direction of the periodic nanostructures and micrometer periodic-like structures could be controlled simply by modifying the polarization direction of the laser beam.

The formation of the periodic nanostructures perpendicular to the laser polarization was consistent with results reported in previous studies [2, 6, 10, 11], and could be attributed to the optical interference of the incident femtosecond laser irradiation with a surface electromagnetic wave. In [17], periodic microstructures on titanium plate were formed using a single beam $800 \mathrm{~nm}$ femtosecond laser pulses in vacuum environment. The orientation of the structures was parallel to the laser polarization, and their period was $1.5 \sim 2.4 \mu \mathrm{m}$, which was similar to our experimental results (see Figs. 6-7).

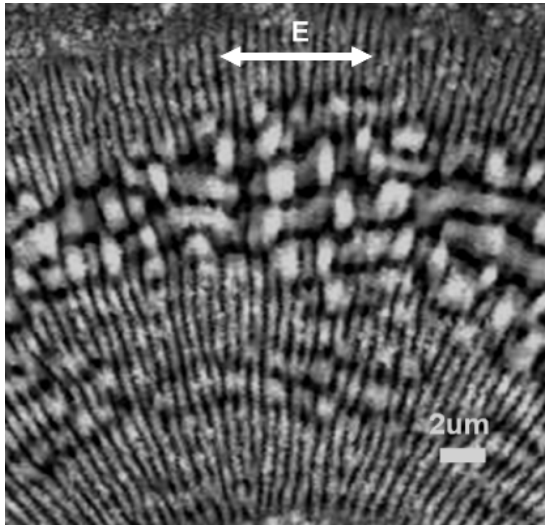

(a)

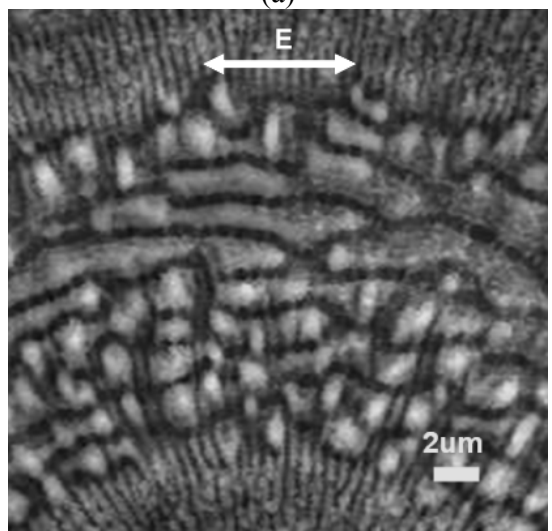

(b)

Fig. 6 Magnified SEM images of point pattern irradiated by azimuthal polarization (a) $0.94 \mathrm{~J} / \mathrm{cm}^{2}$, and (b) $1.01 \mathrm{~J} / \mathrm{cm}^{2}$.

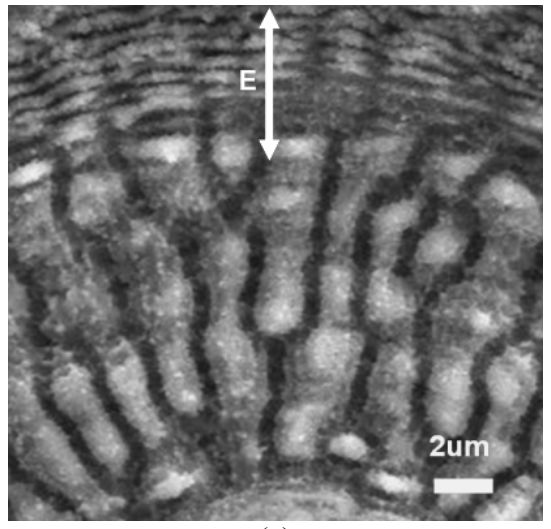

(a)

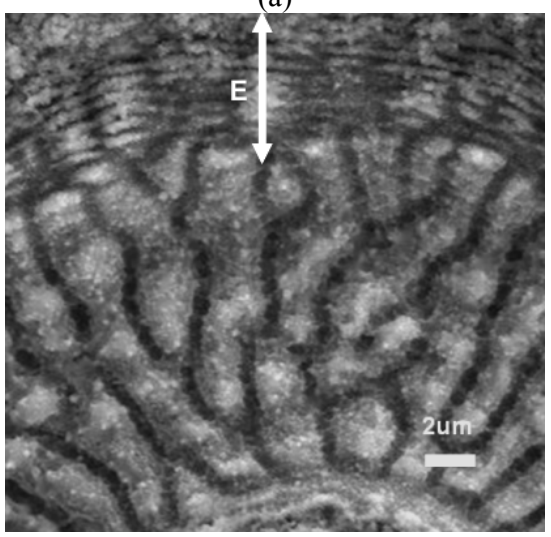

(b)

Fig. 7 Magnified SEM images of point pattern irradiated by radially polarized femtosecond laser beam (a) $0.94 \mathrm{~J} / \mathrm{cm}^{2}$, and (b) $1.01 \mathrm{~J} / \mathrm{cm}^{2}$. 


\section{Conclusions}

This study proposed a novel method for the fabrication of micro/nano structures on silicon wafer. In the proposed approach, the silicon wafer surface was irradiated using a femtosecond laser, under a repetition rate of $1 \mathrm{kHz}$, a wavelength of $800 \mathrm{~nm}$, and radially or azimuthally polarized femtosecond laser beam to create micro/nano structures. The results showed that nanostructures, with a period of around $600 \sim 700 \mathrm{~nm}$, were formed at the low laser fluence region; while micro meter periodic-like structures, with a period of around $2 \sim 3 \mu \mathrm{m}$, were formed at the high laser fluence region. The nanostructures were orientated in a direction perpendicular to the polarization direction, while the micro structures were orientated in a direction parallel to the polarization direction.

\section{Acknowledgements}

The authors appreciate the support of the Ministry of the Economic Affairs (MOEA), Taiwan, R.O.C. in conducting this project.

\section{References}

[1] T.H. Her et al., "Microstructuring of silicon with femtosecond laser pulses," Applied Physics Letters, Vol. 73, 1998.

[2] S. Ameer-Beg et al. "Femtosecond laser microstructuring of materials," Applied Surface Science, Vol. 127$129,1998$.

[3] J. Bonse et al., "Femtosecond laser ablation of siliconmodification thresholds and morphology," Appl. Phys. A, Vol. 74, 2002.

[4] G. Daminelli et al., "Femtosecond laser interaction with silicon under water confinement," Thin Solid Films, Vol. 467, 2004.

[5] R.L. Harzic et al., "Sub-100 nm nanostructuring of silicon by ultra short laser pulses," Optics Express, Vol. 13, 2005.

[6] B. Tan and K. Venkatakrishnan, "A femtosecond laser-induced periodical surface structure on crystalline silicon,” J. Micromech. Microeng., Vol. 16, 2006.
[7] O. Varlamov et al., "Self-organized pattern formation upon femtosecond laser ablation by circularly polarized light," Applied Surface Science, Vol. 252, 2006.

[8] T.H.R. Crawford and H.K. Haugen, "Sub-wavelength surface structures on silicon irradiated by femtosecond laser pulses at 1300 and $2100 \mathrm{~nm}$ wavelengths," Applied Surface Science, Vol. 253, 2007.

[9] V. Zorba et al., "Ultraviolet femtosecond, picosecond and nanosecond laser microstructuring of siliconstructural and optical properties," Applied Optics, Vol. 47, 2008.

[10] T. Takuro et al., "Observation of laser-induced surface waves on flat silicon surface," Applied Physics Letters, Vol. 92, 2008.

[11] J. Bonse et al., "On the role of surface plasmon polaritons in the formation of laser-induced periodic surface structures upon irradiation of silicon by femtosecondlaser pulses," Journal of Applied Physics, Vol. 106, 2009.

[12] M. Schade et al., "High-resolution investigations of ripple structures formed by femtosecond laser irradiation of silicon," Analytical and Bioanalytical Chemistry, Vol. 396, 2010.

[13] S. Sakabe et al., "Mechanism for self-formation of periodic grating structures on a metal surface by a femtosecond laser pulse," Phys. Rev. B, Vol. 79, 2009.

[14] M. Hashida et al., "Ion emission from a metal surface through a multiphoton process and optical field ionization,” Phys. Rev. B, Vol. 81, 2010.

[15]Q. Zhan, "Cylindrical vector beams: from mathematical concepts to applications," Advances in Optics and Photonics Vol. 1, 2009.

[16]D.J. Hwang et al., "Efficiency of silicon micromachining by femtosecond laser pulses in ambient air," Journal of Applied Physics, Vol. 99, 2006.

[17] M. Tsukamoto et al., "Periodic microstructures produced by femtosecond laser irradiation on titanium plate," Vacuum, Vol. 80, 2006.

(Received: June 7 , 2010, Accepted: November 01, 2010) 